\title{
Gene polymorphisms in RANKL/RANK/OPG pathway are associated with ages at menarche and natural menopause in Chinese women
}

\author{
Peng Duan, Zhi-Ming Wang, Jiang Liu, Li-Na Wang, Zhi Yang and Ping Tu*
}

\begin{abstract}
Background: Age at menarche (AAM) and age at natural menopause (AANM) have been shown intimately associated with woman's health later in life. Previous studies have indicated that AAM and AANM are highly heritable. RANKL/RANK/OPG signaling pathway is essential for mammary gland development, which is also found associated with post-menopausal and hormone-related diseases. The aim of this study was to evaluate associations between the polymorphisms in the TNFSF11, TNFRSF11A and TNFRSF11B genes in the RANKL/RANK/OPG pathway with AAM and AANM in Chinese women.

Methods: Post-menopausal Chinese women $(n=845)$ aged from 42 to 89 years were recruited in the study. Information about AAM and AANM were obtained through questionnaires and the genomic DNA was isolated from peripheral blood from the participants. Total 21 tagging single nucleotide polymorphisms (SNPS) of TNFSF11, TNFRSF11A and TNFRSF11B were genotyped.

Results: Three SNPs of TNFRSF11A (rs4500848, rs6567270 and rs1805034) showed significant association with AAM $(P<0.01, P=0.02$ and $P=0.01$, respectively), and one SNP (rs9962159) was significantly associated with AANM $(P=0.03)$. Haplotypes TC and AT (rs6567270-rs 1805034) of TNFRSF11A were found to be significantly associated with AAM ( $P=0.01$ and $P=0.02$, respectively), and haplotypes $G C$ and AC (rs9962159-rs4603673) of TNFRSF11A showed significant association with AANM $(P=0.03$ and $P<0.01$, respectively). No significant association between TNFSF11 or TNFRSF11B gene with AAM or AANM was found.
\end{abstract}

Conclusions: The present study suggests that TNFRSF11A but not TNFSF11 and TNFRSF11B genetic polymorphisms are associated with AAM and AANM in Chinese women. The findings provide evidence that genetic variations in RANKL/ RANK/OPG pathway may be associated with the onset and cessation of the menstruation cycle.

Keywords: RANKL, RANK, OPG, TNFSF11, TNFRSF11A, TNFRSF11B, Polymorphism, Age at menarche, Age at natural menopause

\section{Background}

Age at menarche (AAM) and age at natural menopause (AANM) have been shown intimately associated with woman's health later in life. Women with early menarche have high risks of breast cancer [1], ovarian cancer [2], type 2 diabetes [3] or metabolic syndrome [4], whereas late menarche can increase the risk of osteoporosis [5]. On the other hand, early AANM is associated with

\footnotetext{
* Correspondence: tuping8877@126.com

Department of Endocrinology and Metabolism, Nanchang Key Laboratory of Diabetes, The Third Hospital of Nanchang/The Affiliated Nanchang Hospital of Southern Medical University, NO.2, South Xiangshan road, Nanchang city, Xihu District, Jiangxi province 330009, People's Republic of China
}

increased risk of cardiovascular diseases [6] and osteoporosis [7]. Recent data have shown that AAM and AANM were associated with all-cause mortality [8]. However, the factors that affect AAM and AANM are not entirely clear.

AAM and AANM are complex traits which are influenced by both genetic and environmental factors and their interactions [9]. Twin and familial studies have indicated that AAM and AANM are highly heritable, ranging from $45 \%$ to $74 \%$ for AAM $[10,11]$ and from $49 \%$ to $87 \%$ for AANM $[12,13]$. Genes involved in hormone biosynthesis and metabolic pathways were found to be associated with AAM and AANM $[14,15]$, however, no specific genes have been identified yet. 
The receptor activator of nuclear factor-kappa B ligand (RANKL), its receptor RANK and the decoy receptor osteoprotegerin (OPG) belong to the tumor necrosis factor superfamily and they are encoded by genes TNFSF11, TNFRSF11A and TNFRSF11B, respectively. RANKL/RANK/OPG signaling pathway plays important roles in bone modeling and remodeling [16], cell death and proliferation, inflammation, and immunity $[17,18]$. RANKL/RANK/OPG pathway is also found associated with post-menopausal and hormone-related diseases, such as osteoporosis [19] and reproductive cancer [20]. Furthermore, RANKL is found to be essential for mammary gland development in mice by promoting proliferation and maintaining survival of mammary epithelial cells [21]. Mammary gland changes are one of the hallmarks during menarche and menopause [22,23]. Therefore, RANKL/RANK/OPG pathway may involve in modulating the onset and cessation of the menstrual cycle. The present study investigated the associations of single nucleotide polymorphisms and haplotypes in TNFSF11, TNFRSF11A and TNFRSF11B genes in RANKL/RANK/OPG pathway with AAM and AANM in Chinese females.

\section{Methods}

\section{Participants}

A total 1026 post-menopausal women from ten community centers in Nanchang from December 2011 to December 2012 were enrolled in the study. All the participants were from Han Chinese ethnic group. Age at interview, AAM, AANM, detailed medical history, birth history (number of live delivery), and abortion information (number of abortions) were obtained through a self-designed questionnaire, all the information collected in the study was self-reported. AAM was defined as the age at the first menstrual period. AANM was defined as one year without menstruation after the age at the last menstrual period. For each participant, height $(\mathrm{cm})$ and weight $(\mathrm{kg})$ were measured. The body mass index (BMI) was calculated as weight $/$ height $^{2}$.

All of the participants were subjected to blood counts, liver and kidney function tests, fasting plasma glucose tests. Subjects included in the study had normal blood counts, normal liver and kidney functions and blood glucose levels. Subjects were excluded from the study if they suffered from diseases and surgeries that could affect menstruation, such as severe chronic diseases, rheumatic diseases (e.g. systemic lupus erythematosus, rheumatoid arthritis), severe endocrine and metabolic diseases (e.g. diabetes, hyperparathyroidism, pituitary or adrenal diseases), malabsorption diseases (e.g. chronic diarrhea, anorexia nervosa), cancer, and uterine or ovarian resection. Participants who had taken glucocorticosteroid or sex hormone within the past 3 months were also excluded. Finally, 845 subjects were included in the study. The study was approved by the Ethics Committee of The Third Hospital of Nanchang. Written informed consent was obtained from every participant.

\section{TagSNP selection}

Tagging SNPs of the three genes were selected from the software program Haploview version 4.2 [24] (http://www. broad.mit.edu/mpg/haploview/) with minor allele frequencies $(\mathrm{MAF})>10 \%$ in the Chinese Han population in HapMap (http://www.hapmap.org/), and the pairwise linkage disequilibrium (LD) was greater than a threshold of $r^{2}$ $\left(\mathrm{r}^{2}=0.8\right)$. In addition to, SNPs reported in previous studies or potentially functional SNPs in three candidate genes were forced into the SNP selection process. Finally, a total of 21 SNPs were selected in three genes (9 in TNFRSF11A gene, 6 in TNFSF11 gene, and 6 in TNFRSF11B gene). Of these, 18 SNPs are located in the introns of the three genes, one in $5^{\prime}$-UTR, two in the exonic region. All of these SNPs were authenticated using the NCBI (http://www.ncbi.nlm.nih.gov/SNP/) and HapMap databases.

\section{Genotyping}

Approximately $5 \mathrm{~mL}$ of venous blood was collected from all of the participants after a minimum of $10 \mathrm{~h}$ fasting and stored in tubes containing $100 \mu \mathrm{L}$ of $10 \%$ ethylene diaminetetraacetic acid (EDTA). Genomic DNA was extracted from whole blood samples using the QIAamp DNA Mini Kit (Qiagen Inc., Hilden, Germany). DNA samples concentration and quality were detected spectrophotometrically at $260 / 280 \mathrm{~nm}$ and stored at $-80^{\circ} \mathrm{C}$ until analysed. Genotyping was performed using the highthroughput Sequenom genotyping platform (MassARRAY MALDI-TOF MS system, Sequenom Inc., San Diego, CA). For quality control, $5 \%$ of the samples were repeatedly genotyped, and the results were found to be $100 \%$ concordant.

\section{Statistical analyses}

Genotype frequencies and concordance of the SNPs were analyzed for the Hardy-Weinberg equilibrium (HWE) using the $\chi^{2}$ test. Data were expressed as mean \pm standard deviation. The stepwise multiple regression analysis was used to analyze the relationships between the SNPs and AAM and AANM, subsequently, each SNP with different genotype was analyzed independently using one-way univariate analysis of variance (ANOVA), BMI, age at interview, number of deliveries and abortions were considered as covariates and were adjusted during analysis. Bonferroni correction was used to adjust the $P$ values for multiple comparisons. The statistical analyses were performed using SPSS version 13.0 for Windows (SPSS Inc., Chicago, IL, USA). The linkage disequilibrium structure and allele frequencies were 
examined using Haploview 4.2 software [24]. The significance of each haplotype within the defined blocks was analyzed by PLINK software [25] (http://pngu.mgh. harvard.edu/ purcell/plink/). All analyses were twotailed, and $P$-value $<0.05$ was considered statistically significant.

\section{Results}

\section{Characteristics of the study participants}

The basic characteristics of the 845 participants aged from 42 to 89 years were shown in Table 1 . The mean age at interview was $60.88 \pm 8.72$ years, the mean AAM was $14.97 \pm 2.00$ years and AANM was $48.77 \pm 4.16$ years. No statistically significant association was observed between AAM and AANM $(P=0.15)$.

\section{SNP genotyping and linkage disequilibrium}

The basic characteristics of the SNPs are listed in Table 2. All study SNPs had a minor allele frequency of at least 0.1 and were in agreement with Hardy-Weinberg equilibrium $(P>0.05)$. Linkage disequilibrium between alleles at polymorphic loci was shown in Figure 1. Four haplotype blocks and seventeen of the most common haplotypes (frequency $>5 \%$ ) were further analyzed for the association of haplotype with AAM and AANM.

\section{Association analyses of the SNP and haplotypes with AAM and AANM}

Three SNPs in TNFRSF11A, i.e. rs4500848, rs6567270 and rs1805034, showed significant association with AAM $(P<0.01, P=0.02$ and $P=0.01$, respectively), whereas only rs9962159 in TNFRSF11A was significantly associated with AANM $(P=0.03)$ (Table 2$)$. After correction of age at interview, $\mathrm{BMI}$, number of deliveries and abortions, the associations between those SNPs and AAM or AANM were found significant. After the Bonferroni correction, the rs4500848 was still significantly associated with AAM $(P=0.04)$, however, the associations between the others SNPs with AAM or

Table 1 Characteristics of the $\mathbf{8 4 5}$ participants

\begin{tabular}{lll}
\hline Characteristics & Average & $\mathbf{9 5 \% ~ C l}$ \\
\hline Age (years) & $60.88 \pm 8.72$ & $60.30-61.47$ \\
Age at menarche (years) & $14.97 \pm 2.00$ & $14.83-15.10$ \\
Age at menopause (years) & $48.77 \pm 4.16$ & $48.49-49.05$ \\
Height $(\mathrm{cm})$ & $154.18 \pm 5.97$ & $153.78-154.58$ \\
Weight $(\mathrm{kg})$ & $58.21 \pm 8.73$ & $57.62-58.80$ \\
BMI $\left(\mathrm{kg} / \mathrm{m}^{2}\right)$ & $24.46 \pm 3.20$ & $24.25-24.68$ \\
Number of deliveries & $2.14 \pm 1.32$ & $2.05-2.23$ \\
Number of spontaneous abortions & $0.13 \pm 0.44$ & $0.10-0.16$ \\
Number of induced abortions & $1.21 \pm 1.27$ & $1.13-1.30$ \\
\hline
\end{tabular}

The data are presented as the means \pm standard deviation. BMI, body mass index. $\mathrm{Cl}$, confidence interval.
AANM were no longer statistically significant (Table 2). Individuals with the T/T genotype of SNP rs 4500848 had an earlier onset of menarche by 0.59 years than did those with the $\mathrm{C} / \mathrm{C}$ genotype. Likewise, women with the G/G genotype of SNP $r s 9962159$ had an earlier menopause by 0.79 years than those with the A/A genotype (Table 3 ).

Two haplotypes (TC and AT) of block rs6567270rs1805034 of TNFRSF11A were found significantly associated with $\mathrm{AAM}(P=0.01$ and $P=0.02$, respectively) (Table 4). Haplotypes GC and AC of block rs9962159rs4603673 of TNFRSF11A were significantly associated with AANM $(P=0.03$ and $P<0.01$, respectively). Haplotype TAGCGT of block rs9525641-rs2277439rs2324851-rs2875459-rs2200287-rs9533166 of TNFSF11 showed marginally significant association with AAM $(P=0.06)$. Notably, all the significantly associated SNPs and haplotypes were observed in TNFRSF11A. SNPs and haplotypes in TNFSF11 and TNFRSF11B genes did not show significant association with either AAM or AANM.

\section{Discussion}

According to the previous studies, there was a direct relationship between AAM and AANM, women with earlier menarche had earlier menopause in Poland [26]. However, other studies had reported no association between AAM and AANM [27]. In this tudy, no statistically significant association was observed between AAM and AANM. The present study revealed that three SNPs ( $r s 4500848, r s 6567270$ and $r s 1805034)$ and two haplotypes of TNFRSF11A showed significant association with AAM in Chinese women. These findings are in line to a previous report by Pan et al. [28], the authors found five SNPs (rs7239261, rs8094884, rs3826620, rs8089829, and $r s 9956850)$ and seven haplotypes of TNFRSF11A significantly associated with AAM in Chinese women. Thus, polymorphisms in TNFRSF11A are highly associated with AAM in Chinese women. In contrast to TNFRSF11A, SNPs of TNFSF11 did not show association with AAM in our study. Noticeably, two SNPs (rs9525641 and $r$ 2200287) of TNFSF11 displayed a strong association with AAM in white women [29], but no significant association was observed between the two SNPs and AAM in our study. The inconsistency between the results of the present study and the other [29] may due to the different ethnic populations used, different sample sizes and statistical approaches. The inconsistent results were also observed in the association of TNFSF11 gene polymorphisms and AANM. In the present study, no significant association between polymorphisms of TNFSF11 and AANM was found in Chinese women, however, such relationship was reported in white women, two SNPs (rs346578 and rs9525641) of TNFSF11 showed association with AANM [29]. Regardless of the discrepancy in TNFSF11, we and the others [29] both 
Table 2 Associations for the SNPs of TNFSF11, TNFRSF11A and TNFRSF11B genes with AAM and AANM

\begin{tabular}{|c|c|c|c|c|c|c|c|c|c|c|c|}
\hline \multirow[t]{2}{*}{ Gene } & \multirow[t]{2}{*}{ SNP } & \multirow[t]{2}{*}{ Allele } & \multirow[t]{2}{*}{ Function } & \multirow[t]{2}{*}{ HWE } & \multirow[t]{2}{*}{ MAF } & \multicolumn{3}{|l|}{ AAM } & \multicolumn{3}{|l|}{ AANM } \\
\hline & & & & & & Beta & $P$ & $P$-Bonf & Beta & $P$ & P-Bonf \\
\hline TNFRSF11B & rs 1485286 & $\mathrm{C} / T$ & Intron & 0.4237 & $T=0.406$ & 0.0697 & 0.49 & 1.00 & 0.2428 & 0.25 & 1.00 \\
\hline TNFRSF11B & rs11573869 & $A / G$ & Intron & 0.8783 & $G=0.165$ & -0.0529 & 0.69 & 1.00 & 0.1109 & 0.68 & 1.00 \\
\hline TNFRSF11B & rs3102728 & $T / C$ & Intron & 0.2913 & $C=0.138$ & 0.0726 & 0.61 & 1.00 & -0.2352 & 0.43 & 1.00 \\
\hline TNFRSF11B & rs11573819 & $G / A$ & Intron & 0.9402 & $A=0.157$ & 0.0501 & 0.71 & 1.00 & -0.1709 & 0.54 & 1.00 \\
\hline TNFRSF11B & rs2073618 & $C / G$ & Asn by Lys & 0.5148 & $G=0.258$ & -0.0065 & 0.95 & 1.00 & 0.2687 & 0.25 & 1.00 \\
\hline TNFRSF11B & rs2073617 & $A / G$ & UTR-5 & 0.7887 & $G=0.382$ & -0.1137 & 0.26 & 1.00 & 0.1741 & 0.41 & 1.00 \\
\hline TNFSF11 & rs9525641 & $T / C$ & Intron & 0.1546 & $C=0.472$ & 0.0810 & 0.42 & 1.00 & -0.0656 & 0.75 & 1.00 \\
\hline TNFSF11 & rs 2277439 & $A / G$ & Intron & 0.5905 & $G=0.295$ & -0.0611 & 0.56 & 1.00 & 0.2430 & 0.27 & 1.00 \\
\hline TNFSF11 & rs 2324851 & $G / A$ & Intron & 0.6728 & $A=0.294$ & -0.0699 & 0.51 & 1.00 & 0.2351 & 0.29 & 1.00 \\
\hline TNFSF11 & rs 2875459 & $C / T$ & Intron & 0.8542 & $\mathrm{~T}=0.220$ & -0.1036 & 0.38 & 1.00 & -0.0189 & 0.94 & 1.00 \\
\hline TNFSF11 & rs2200287 & $G / A$ & Intron & 0.8891 & $A=0.220$ & -0.0898 & 0.44 & 1.00 & -0.0215 & 0.94 & 1.00 \\
\hline TNFSF11 & rs9533166 & $T / C$ & Intron & 0.5125 & $C=0.131$ & -0.1694 & 0.23 & 1.00 & -0.2154 & 0.47 & 1.00 \\
\hline TNFRSF11A & rs9962159 & $A / G$ & Intron & 0.4172 & $G=0.435$ & 0.1338 & 0.17 & 1.00 & -0.4434 & 0.03 & 0.57 \\
\hline TNFRSF11A & rs4603673 & $C / G$ & Intron & 0.7190 & $G=0.162$ & -0.1363 & 0.31 & 1.00 & -0.2551 & 0.36 & 1.00 \\
\hline TNFRSF11A & rs7239261 & $C / A$ & Intron & 0.2342 & $A=0.239$ & -0.1656 & 0.14 & 1.00 & 0.3123 & 0.18 & 1.00 \\
\hline TNFRSF11A & rs4500848 & $C / T$ & Intron & 0.7267 & $T=0.262$ & -0.3395 & $<0.01$ & 0.04 & -0.0378 & 0.87 & 1.00 \\
\hline TNFRSF11A & rs6567270 & $T / A$ & Intron & 0.1116 & $A=0.408$ & 0.2265 & 0.02 & 0.39 & 0.1197 & 0.55 & 1.00 \\
\hline TNFRSF11A & rs 1805034 & $T / C$ & Ala by Val & 0.3758 & $C=0.288$ & -0.2790 & 0.01 & 0.22 & 0.0069 & 0.98 & 1.00 \\
\hline TNFRSF11A & rs4303637 & $\mathrm{C} / T$ & Intron & 0.9818 & $C=0.471$ & 0.1618 & 0.10 & 1.00 & 0.0701 & 0.73 & 1.00 \\
\hline TNFRSF11A & rs4941131 & $T / C$ & Intron & 0.8166 & $C=0.330$ & 0.0437 & 0.67 & 1.00 & -0.1892 & 0.38 & 1.00 \\
\hline TNFRSF11A & rs9646629 & $G / C$ & Intron & 0.2416 & $C=0.460$ & 0.1461 & 0.14 & 1.00 & -0.2256 & 0.28 & 1.00 \\
\hline
\end{tabular}

HWE, $P$ values for Hardy-Weinberg equilibrium. MAF, minor allele frequency. Beta, the regression coefficient. $P$-Bonf, $P$-value by Bonferroni correction. AAM, age at menarche. AANM, age at natural menopause.

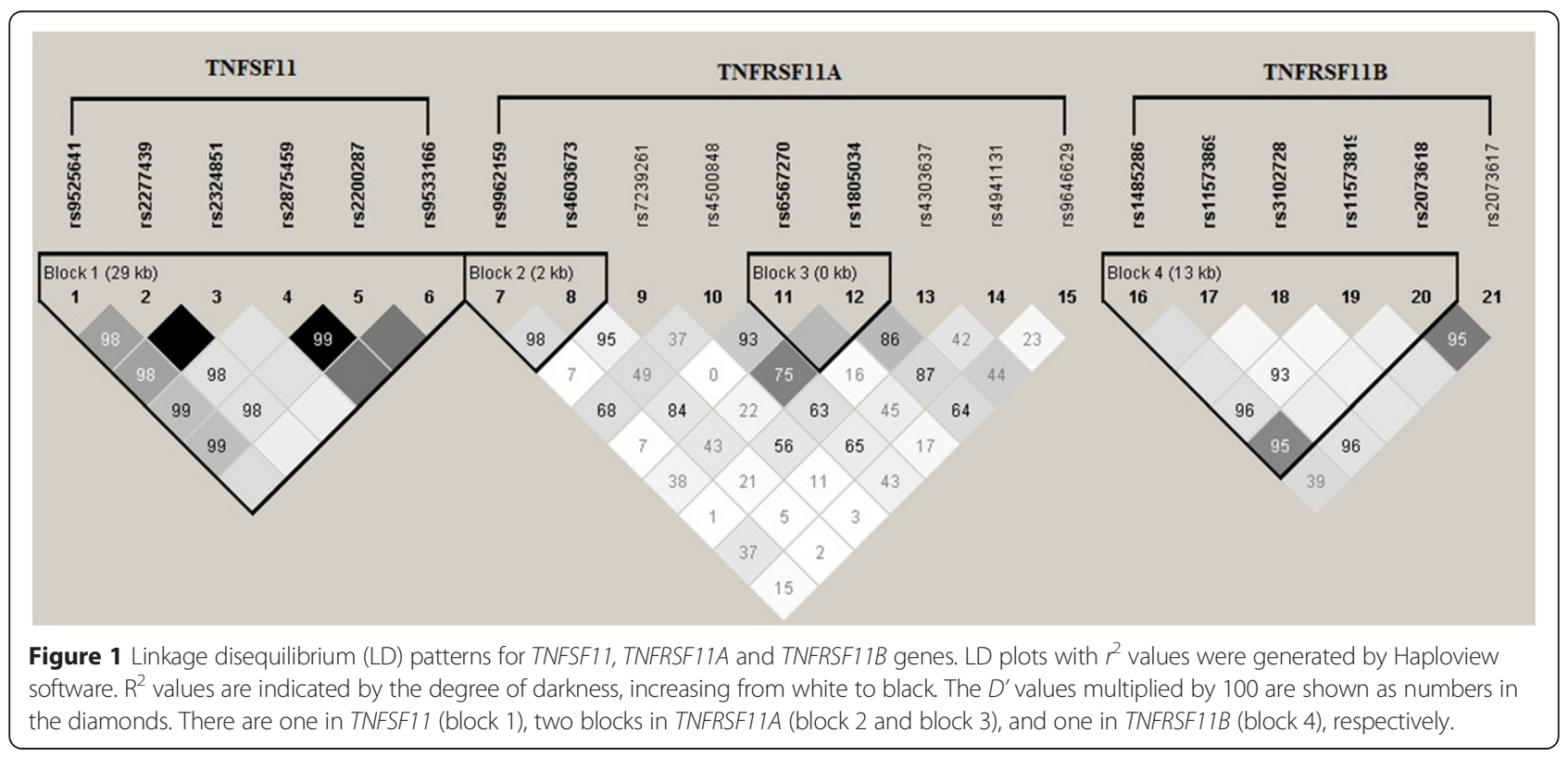


Table 3 Significant associations for the single SNPs of TNFSF11, TNFRSF11A and TNFRSF11B genes with AAM and AANM

\begin{tabular}{llllll}
\hline Genotype & $\mathbf{n}$ & AAM & Genotype & $\mathbf{n}$ & AAM \\
\hline rs4500848 & & & rs/805034 & & \\
$C / C$ & 471 & $15.16 \pm 1.95$ & $C / C$ & 64 & $14.53 \pm 2.17$ \\
$C / T$ & 313 & $14.76 \pm 2.07$ & $C / T$ & 358 & $14.86 \pm 2.04$ \\
$T / T$ & 61 & $14.57 \pm 1.85$ & $T / T$ & 423 & $15.12 \pm 1.93$ \\
$P$-value & & $<0.01$ & $P$-value & & 0.04 \\
\hline Genotype & $\mathbf{n}$ & AAM & Genotype & $\mathbf{n}$ & AANM \\
\hline rs6567270 & & & rs9962159 & & \\
A $/ A$ & 152 & $15.35 \pm 2.07$ & G/G & 166 & $48.45 \pm 4.18$ \\
A T & 385 & $14.92 \pm 2.02$ & G/A & 403 & $48.56 \pm 4.23$ \\
$T / T$ & 308 & $14.84 \pm 1.91$ & A $/ A$ & 276 & $49.26 \pm 4.00$ \\
$P$-value & & 0.03 & $P$-value & & 0.05 \\
\hline
\end{tabular}

found a strong association between polymorphisms of TNFRSF11A and AANM.

The associations between polymorphisms of TNFRSF11A with AAM and AANM found in the present study can be explained by its possible roles in

Table 4 The associations of haplotypes of TNFSF11, TNFRSF11A and TNFRSF11B genes with AAM and AANM

\begin{tabular}{|c|c|c|c|c|c|c|}
\hline \multirow[t]{2}{*}{ Gene } & \multirow[t]{2}{*}{ Haplotype } & \multirow[t]{2}{*}{ Frequency } & \multicolumn{2}{|l|}{ AAM } & \multicolumn{2}{|l|}{ AANM } \\
\hline & & & Beta & $P$ & Beta & $P$ \\
\hline \multicolumn{7}{|c|}{ TNFSF11: rs9525641-rs2277439-rs2324851-rs2875459-rs2200287-rs9533166 } \\
\hline & TAGTAC & 0.131 & -0.1686 & 0.24 & -0.2171 & 0.46 \\
\hline & TAGTAT & 0.088 & 0.0148 & 0.93 & 0.2392 & 0.50 \\
\hline & TGACGT & 0.292 & -0.0737 & 0.49 & 0.1958 & 0.38 \\
\hline & CAGCGT & 0.469 & 0.0773 & 0.44 & -0.1007 & 0.63 \\
\hline & TAGCGT & 0.017 & 0.7854 & 0.06 & -1.3300 & 0.09 \\
\hline \multicolumn{7}{|c|}{ TNFRSF11A: rs9962159-rs4603673 } \\
\hline & $A G$ & 0.161 & -0.1455 & 0.28 & -0.2432 & 0.38 \\
\hline & GC & 0.434 & 0.1294 & 0.18 & -0.4383 & 0.03 \\
\hline & $A C$ & 0.405 & -0.0578 & 0.55 & 0.5747 & $<0.01$ \\
\hline \multicolumn{7}{|c|}{ TNFRSF11A : rs6567270-rs 1805034} \\
\hline & $T C$ & 0.288 & -0.2787 & 0.01 & 0.0069 & 0.98 \\
\hline & $A T$ & 0.408 & 0.2263 & 0.02 & 0.1197 & 0.55 \\
\hline & $\pi$ & 0.305 & -0.0113 & 0.91 & -0.1408 & 0.51 \\
\hline \multicolumn{7}{|c|}{ TNFRSF11B : rs 1485286-rs11573869-rs3102728-rs11573819-rs2073618 } \\
\hline & TATGG & 0.250 & 0.0311 & 0.78 & 0.2791 & 0.24 \\
\hline & CATAC & 0.155 & 0.0770 & 0.57 & -0.1808 & 0.52 \\
\hline & CACGC & 0.139 & 0.0726 & 0.61 & -0.2352 & 0.43 \\
\hline & CGTGC & 0.164 & -0.0407 & 0.76 & 0.1067 & 0.70 \\
\hline & TATGC & 0.154 & 0.0955 & 0.48 & 0.0410 & 0.88 \\
\hline & CATGC & 0.130 & -0.2041 & 0.17 & -0.1828 & 0.56 \\
\hline
\end{tabular}

The analyses were performed under an additive model adjusted for age at interview and BMI. Beta, regression coefficient. mammary gland development and menstruation. First, TNFRSF11A belongs to RANKL/RANK/OPG signaling pathway. RANKL plays an important role in mammary gland development, indicating its potential role in regulating or responding to sex hormone fluctuation and subsequently influencing menstrual cycles. Studies have shown that gonadotropin-releasing hormone (GnRH) can modulate RANKL expression in breast cancer cells [30], and expressions of RANK and RANKL in different cell lines are controlled by estrogen [31], follicle-stimulating hormone [32], and dehydroepiandrosterone [33]. Estrogen is also found to regulate gene expression and ratio of the RANKL/OPG [34]. Second, RANKL signaling pathway can stimulate ductal side-branching and alveologenesis in the mammary gland in mouse [35]. RANKL can be induced in mammary epithelium and can regulate the proliferation of cells [36]. Therefore, RANK signaling pathway may influence onset of puberty and menstrual cycle by regulating mammary gland development. Third, genome-wide association (GWA) studies have identified some novel genetic loci associated with AAM and AANM [37,38]. Gene set enrichment pathway analyses using the GWA dataset found that nuclear factor-kappa B (NF-kB) signaling pathway may be associated with timing of menopause [39]. Recent studies have revealed that NF- $\mathrm{KB}$ pathway plays an important role in mammary ductal morphogenesis [40], and ovarian cell function in animals [41]. It was well established that the RANKL/RANK/OPG pathway can activate NF- $\kappa B$ and its downstream players [42]. Thus, genes (e.g. TNFRSF11A) in the RANKL/RANK/OPG pathway may have role in the onset and cessation of the menstruation cycle.

The present study has some limitations. First, beside genetic other factors can influence timing of menarche and menopause, e.g. environment and socioeconomic status. We studied only the relationship between genetic variations and AAM and AANM. Furthermore, geneenvironment interactions may also play a role in causing variation in the AAM and AANM. Second, The data of AAM and AANM were collected through retrospective self-report, which may cause recall bias. The participants in the study were aged from 42 to 89 years, with long interval periods, which might potentially incur recall error. It is reported that the accuracy of long-term recall of AAM and AANM varied from $70 \%$ to $84 \%[43,44]$. In fact, it was found that some participants could not remember the exact age at the first menstrual period, and those subjects were excluded from the study. Large-scale studies are needed to confirm current findings, and the precise mechanisms underlying the observed associations in our study remain to be determined.

\section{Conclusions}

The present study, for the first time, demonstrated that TNFRSF11A but not TNFSF11 and TNFRSF11B genetic 
polymorphisms are associated with AAM and AANM in Chinese women. The findings provide evidence that genetic variations in RANKL/RANK/OPG pathway may be associated with the onset and cessation of the menstruation cycle.

\section{Abbreviations}

AAM: Age at menarche; AANM: Age at natural menopause:

OPG: Osteoprotegerin; RANK: Receptor activator of nuclear factor-kappa B; RANKL: Receptor activator of nuclear factor-kappa B ligand; SNP: Single nucleotide polymorphisms; BMI: Body mass index; HWE: Hardy-Weinberg equilibrium; GnRH: Gonadotropin-releasing hormone; GWA: Genome-wide association; NF-KB: Nuclear factor-kappa B.

\section{Competing interests}

The authors declare that they have no competing interests.

\section{Authors' contributions}

PD participated in the molecular genetic studies, conducted statistical analyses and drafted the manuscript. ZMW assisted in genetic studies and drafted the manuscript. $J L$ and LNW collected all samples and questionnaire information. ZY helped in recruiting subjects and statistical analyses. PT conceived of the study, participated in its design, recruited participants, revised the manuscript. All authors read and approved the final manuscript.

\section{Acknowledgements}

The authors would like to thank Prof. Gao Xin Yuan and Dr. Zhang Zeng for technical assistance. We thank all the participants in this study. This study was supported by grants from the National Natural Science Foundation of China (no. 81260133) and Key Projects of Health Department of Jiangxi province, China (no. 20114030).

\section{Received: 5 December 2014 Accepted: 1 April 2015}

\section{Published online: 13 April 2015}

\section{References}

1. Ritte R, Lukanova A, Tjønneland A, Olsen A, Overvad K, Mesrine S, et al. Height, age at menarche and risk of hormone receptor -positive and -negative breast cancer: a cohort study. Int J Cancer. 2013;132:2619-29.

2. Gong TT, Wu QJ, Vogtmann E, Lin B, Wang YL. Age at menarche and risk of ovarian cancer: a meta-analysis of epidemiological studies. Int J Cancer. 2013;132:2894-900.

3. Stöckl D, Döring A, Peters A, Thorand B, Heier M, Huth C, et al. Age at menarche is associated with prediabetes and diabetes in women (aged 32-81 years) from the general population: the KORA F4 study. Diabetologia. 2012;55:681-8.

4. Akter S, Jesmin S, Islam M, Sultana SN, Okazaki O, Hiroe M, et al. Association of age at menarche with metabolic syndrome and its components in rural Bangladeshi women. Nutr Metab (Lond). 2012;9:99.

5. Eastell R. Role of oestrogen in the regulation of bone turnover at the menarche. J Endocrinol. 2005;185:223-34.

6. Cui R, Iso H, Toyoshima H, Date C, Yamamoto A, Kikuchi S, et al. Relationships of age at menarche and menopause, and reproductive year with mortality from cardiovascular disease in Japanese postmenopausal women: the JACC study. J Epidemiol. 2006;16:177-84.

7. Sioka C, Fotopoulos A, Georgiou A, Xourgia X, Papadopoulos A, Kalef-Ezra JA. Age at menarche, age at menopause and duration of fertility as risk factors for osteoporosis. Climacteric. 2010;13:63-71.

8. Li S, Rosenberg L, Wise LA, Boggs DA, LaValley M, Palmer JR. Age at natural menopause in relation to all-cause and cause-specific mortality in a follow-up study of US black women. Maturitas. 2013;75:246-52.

9. Dvornyk V. Waqar-ul-Haq: genetics of age at menarche: a systematic review. Hum Reprod Update. 2012;18:198-210.

10. Snieder H, MacGregor AJ, Spector TD. Genes control the cessation of a woman's reproductive life: a twin study of hysterectomy and age at menopause. J Clin Endocrinol Metab. 1998:83:1875-80.

11. Sharma K. Genetic basis of human female pelvic morphology: a twin study. Am J Phys Anthropol. 2002;117:327-33.

12. Murabito JM, Yang Q, Fox C, Wilson PW, Cupples LA. Heritability of age at natural menopause in the Framingham heart study. J Clin Endocrinol Metab. 2005;90:3427-30.
13. de Bruin JP, Bovenhuis $H$, van Noord PA, Pearson PL, van Arendonk JA, te Velde $E R$, et al. The role of genetic factors in age at natural menopause. Hum Reprod. 2001;16:2014-8.

14. He C, Kraft P, Chen C, Buring JE, Paré G, Hankinson SE, et al. Genome-wide association studies identify loci associated with age at menarche and age at natural menopause. Nat Genet. 2009;41:724-8.

15. Perry JR, Hsu YH, Chasman DI, Johnson AD, Elks C, Albrecht E, et al. DNA mismatch repair gene $\mathrm{MSH} 6$ implicated in determining age at natural menopause. Hum Mol Genet. 2014;23:2490-7.

16. Wada T, Nakashima T, Hiroshi N, Penninger JM. RANKL-RANK signaling in osteoclastogenesis and bone disease. Trends Mol Med. 2006;12:17-25.

17. Boyce BF, Xing L. Biology of RANK, RANKL, and osteoprotegerin. Arthritis Res Ther. 2007;9 Suppl 1:S1.

18. Hanada R, Hanada T, Sigl V, Schramek D, Penninger JM. RANKL/RANK- beyond bones. J Mol Med (Berl). 2011;89:647-56.

19. Rinotas V, Niti A, Dacquin R, Bonnet N, Stolina M, Han CY, et al. Novel genetic models of osteoporosis by overexpression of human RANKL in transgenic mice. J Bone Miner Res. 2014;29:1158-69.

20. Lane D, Matte I, Laplante C, Garde-Granger P, Rancourt C, Piché A. Osteoprotegerin (OPG) activates integrin, focal adhesion kinase (FAK), and Akt signaling in ovarian cancer cells to attenuate TRAlL-induced apoptosis. J Ovarian Res. 2013;6:82.

21. Cao Y, Bonizzi G, Seagroves TN, Greten FR, Johnson R, Schmidt EV, et al. IKKalpha provides an essential link between RANK signaling and cyclin D1 expression during mammary gland development. Cell. 2001;107:763-75.

22. Karapanou O, Papadimitriou A. Determinants of menarche. Reprod Biol Endocrinol. 2010;8:115.

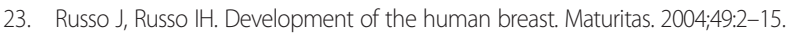

24. Barrett JC, Fry B, Maller J, Daly MJ. Haploview: analysis and visualization of LD and haplotype maps. Bioinformatics. 2005;21:263-5.

25. Purcell S, Neale B, Todd-Brown K, Thomas L, Ferreira MA, Bender D, et al. PLINK: a tool set for whole-genome association and population-based linkage analyses. Am J Hum Genet. 2007;81:559-75.

26. Kaczmarek M. The timing of natural menopause in Poland and associated factors. Maturitas. 2007:57:139-53.

27. Otero UB, Chor D, Carvalho MS, Faerstein E, Lopes Cde S, Werneck GL. Lack of association between age at menarche and age at menopause: Pró-Saúde Study, Rio de Janeiro, Brazil. Maturitas. 2010;67:245-50.

28. Pan R, Liu YZ, Deng HW, Dvornyk V. Association analyses suggest the effects of RANK and RANKL on age at menarche in Chinese women. Climacteric. 2012;15:75-81.

29. Lu Y, Liu P, Recker RR, Deng HW, Dvornyk V. TNFRSF11A and TNFSF11 are associated with age at menarche and natural menopause in white women. Menopause. 2010;17:1048-54.

30. Schubert A, Schulz H, Emons G, Grundker C. Expression of osteoprotegerin and receptor activator of nuclear factor-KB ligand (RANKL) in HCC70 breast cancer cells and effects of treatment with gonadotropin-releasing hormone on RANKL expression. Gynecol Endocrinol. 2008;24:331-8.

31. Bord S, Ireland DC, Beavan SR, Compston JE. The effects of estrogen on osteoprotegerin, RANKL, and estrogen receptor expression in human osteoblasts. Bone. 2003;32:136-41.

32. Cannon JG, Kraj B, Sloan G. Follicle-stimulating hormone promotes RANK expression on human monocytes. Cytokine. 2011:53:141-4.

33. Wang YD, Tao MF, Wang L, Cheng WW, Wan XP. Selective regulation of osteoblastic OPG and RANKL by dehydroepiandrosterone through activation of the estrogen receptor $\beta$-mediated MAPK signaling pathway. Horm Metab Res. 2012;44:494-500.

34. Bashir A, Mak YT, Sankaralingam S, Cheung J, McGowan NW, Grigoriadis AE, et al. Changes in RANKL/OPG/RANK gene expression in peripheral mononuclear cells following treatment with estrogen or raloxifene. Steroids. 2005;70:847-55.

35. Fernandez-Valdivia R, Mukherjee A, Ying Y, Li J, Paquet M, DeMayo FJ, et al. The RANKL signaling axis is sufficient to elicit ductal side-branching and alveologenesis in the mammary gland of the virgin mouse. Dev Biol. 2009;328:127-39.

36. Kim NS, Kim HJ, Koo BK, Kwon MC, Kim YW, Cho Y, et al. Receptor activator of NF-kappaB ligand regulates the proliferation of mammary epithelial cells via Id2. Mol Cell Biol. 2006;26:1002-13.

37. Delahanty RJ, Beeghly-Fadiel A, Long JR, Gao YT, Lu W, Xiang YB, et al. Evaluation of GWAS-identified genetic variants for age at menarche among Chinese women. Hum Reprod. 2013;28:1135-43. 
38. Pyun JA, Kim S, Cho NH, Koh I, Lee JY, Shin C, et al. Genome-wide association studies and epistasis analyses of candidate genes related to age at menarche and age at natural menopause in a Korean population. Menopause. 2014;21:522-9.

39. Stolk L, Perry JR, Chasman DI, He C, Mangino M, Sulem P, et al. Meta-analyses identify 13 loci associated with age at menopause and highlight DNA repair and immune pathways. Nat Genet. 2012;44:260-8.

40. Voutilainen $\mathrm{M}$, Lindfors $\mathrm{PH}$, Lefebvre $\mathrm{S}$, Ahtiainen L, Fliniaux I, Rysti $\mathrm{E}$, et al. Ectodysplasin regulates hormone-independent mammary ductal morphogenesis via NF-kB. Proc Natl Acad Sci U S A. 2012;109:5744-9.

41. Pavlová S, Klucska K, Vašiček D, Ryban L, Harrath AH, Alwasel SH, et al. The involvement of SIRT1 and transcription factor NF-KB (p50/p65) in regulation of porcine ovarian cell function. Anim Reprod Sci. 2013;140:180-8.

42. Hanada R, Hanada T, Penninger JM. Physiology and pathophysiology of the RANKL/RANK system. Biol Chem. 2010;391:1365-70.

43. Must A, Phillips SM, Naumova EN, Blum M, Harris S, Dawson-Hughes B, et al. Recall of early menstrual history and menarcheal body size: after 30 years, how well do women remember? Am J Epidemiol. 2002;155:672-9.

44. den Tonkelaar I. Validity and reproducibility of self-reported age at menopause in women participating in the DOM-project. Maturitas. 1997;27:117-23.

\section{Submit your next manuscript to BioMed Central and take full advantage of:}

- Convenient online submission

- Thorough peer review

- No space constraints or color figure charges

- Immediate publication on acceptance

- Inclusion in PubMed, CAS, Scopus and Google Scholar

- Research which is freely available for redistribution 